\title{
Avaliação da Qualidade e Satisfação de Vida dos Pacientes antes da Internação na Unidade de Terapia Intensiva e após a Alta Hospitalar ${ }^{\star}$
}

\author{
Evaluation the Quality and Satisfaction of Life of Patients, \\ before Admission in Intensive Care Unit and after Hospital Discharge.
}

Fernando Osni Machado ${ }^{2}$, Geovana Basso², Cleyton de Souza Margarida², Rachel Duarte Moritz ${ }^{3}$

RESUMO

JUSTIFICATIVA E OBJETIVOS: Analisar a qualidade e satisfação de vida (QSV) dos pacientes antes da internação na UTI e após a alta hospitalar. O objetivo deste estudo foi verificar se as características demográficas, clínicas e terapêuticas interferiram nessa QSV.

MÉTODO: Foi realizado um estudo do tipo coorte prospectivo, com abordagem quanti-qualitativa. Foram analisados os pacientes internados na UTI do Hospital Universitário da Universidade Federal de Santa Catarina, por mais de 24 horas, entre os meses de abril e julho de 2005. Coletaram-se dados referentes a QSV dos pacientes antes da internação na UTI, assim como suas características demográficas, clínicas e terapêuticas. Posteriormente, aplicou-se, por telefone, 7, 90 e 180 dias após a alta hospitalar, um questionário que avaliou os dados sobre a QSV dos pacientes; subdivididos em 2 grupos: QSV melhor/inalterada e piora

1. Doutor em Medicina (USP); Mestre em Medicina Interna (UFSC); Professor do Departamento de Clínica Médica da UFSC.

2. Graduando da UFSC.

3. Doutora em Engenharia de Produção - Ergonomia (UFSC); Mestre em Ciências Médicas (UFSC); Professora do Departamento de Clínica Médica (UFSC).

${ }^{*}$ Recebido do Hospital Universitário Professor Polydoro Ernani de São Thiago da Universidade Federal de Santa Catarina (UFSC), Florianópolis, SC.

Apresentado em 06 de setembro de 2006

Aceito para publicação em 01 de fevereiro de 2007

Endereço para correspondência:

Prof. Dr. Fernando Osni Machado

Rua Antônio Carlos Ferreira, 238 - Agronômica

88025-210 Florianópolis, SC

Fone: (048) 9980-9389

E-mail: fernandoo.machado@gmail.com

(C)Associação de Medicina Intensiva Brasileira, 2007 da QSV. Para a análise estatística foram utilizados os testes $t$ de Student e Qui-quadrado. Foi considerado significativo $\leq 0,05$.

RESULTADOS: Sessenta e oito pacientes preencheram os critérios de admissão, 21 completaram o estudo. A comparação entre a QSV de 7, 90 e 180 dias após a alta hospitalar revelou que a qualidade de vida (QV) dos pacientes melhorou e que 90,5\% estavam satisfeitos. Os pacientes cuja QV foi melhor/inalterada foram os mais idosos. Os grupos foram semelhantes, em relação ao sexo, escolaridade, escore APACHE ॥ médio, tempo de internação, uso de ventilação mecânica e fármaco vasoativo. Sessenta por cento dos pacientes havia retornado ao trabalho após 180 dias.

CONCLUSÕES: Houve uma tendência de os pacientes sentirem-se satisfeitos após a alta hospitalar, bem como a melhora da qualidade de vida com o decorrer do tempo. Mesmo aqueles que referiram piora, encontravamse bem para retomar suas atividades laborativas.

Unitermos: Medicina Intensiva, Qualidade de Vida, Tratamento intensivo

\section{SUMMARY}

BACKGROUND AND OBJETIVES: To evaluate the quality and satisfaction of life (QSL) of patients before admission in ICU, and after hospital discharge. To verify the influence of the patient's demographic/clinic/therapeutic factors in the QSL.

METHODS: Prospective cohort study with qualiquantitative approach. All patients admitted in ICU/ HU/UFSC from April-July 2005, who's stayed more than 24 hours were included. Initially, the data of QSL before ICU admission, patient's demographics/clinics/therapeutics features were recorded. Afterwards, by telephone, 7, 90 and 180 days after hospital discharge, the patients answered the questionnaires 
about QSL. In the sequence, all patients were subdivided into 2 main groups: unchanged or better, and worse QSL. Data were analyzed using t Student and Chi-square tests ( $p$-value $\leq 0.05)$.

RESULTS: Sixty eight patients were enrolled into the study. Completed questionnaires were obtained from 21 of them. A comparison of 7, 90 and 180 days after hospital discharge showed that QSL of patients was unchanged or better at 90 and 180 days. The majority of patients expressed more satisfaction in that moment. Unchanged or better QSL was associated with advanced age. However, there were no statistical significant differences in sex, schooling, APACHE II score, length of stay, mechanical ventilation and used drugs. Sixty percent returned to their previous work.

CONCLUSIONS: There was a tendency for patients who felt themselves satisfied after hospital discharge to have their QSL improved as time went bye. Better QSL was associated with advanced age. Even when patients reported worse QSL they returned to their previous work.

Key Words: ICU treatment, Intensive Care Medicine, Quality of life

\section{INTRODUÇÃO}

Após a segunda metade do século XX, mais especificamente após a Segunda Grande Guerra, o desenvolvimento tecnológico foi exponencial. Em decorrência desse fato houve um crescente aperfeiçoamento do tratamento nas UTI, o que tem gerado um novo paradigma, já que em muitas ocasiões, o aperfeiçoamento tecnológico tem levado ao prolongamento do processo de morrer do paciente gravemente enfermo, fato denominado de obstinação terapêutica. A obstinação terapêutica tem gerado a necessidade de o médico intensivista decidir sobre a recusa ou a suspensão de tratamentos considerados fúteis ou inúteis, o que torna necessária a reformulação quanto às metas dos cuidados em UTI, partindo-se, muitas vezes, da cura para o conforto ${ }^{1-3}$. Tendo em vista a otimização da utilização dos leitos nas UTI tem sido desenvolvida e cada vez mais são utilizados os índices prognósticos e critérios específicos de internação e alta nessas unidades $^{4-6}$

Diante dessa realidade, tem havido um interesse crescente da avaliação sobre a qualidade e a satisfação de vida (QSV) do paciente que tenha recebido alta da UTI. Essa avaliação poderá ser importante para futuras tomadas de decisão sobre internações nessas unidades e para a análise da sua eficácia. Tem sido demonstrado que os pacientes com boa qualidade de vida pré-internação na UTI têm maior possibilidade de recuperação. Ao contrário, a falência de múltiplos órgãos e o êxito letal está relacionada à baixa qualidade de vida pré-internação na UTI ${ }^{7-10}$. Questionários têm sido elaborados para a avaliação da QSV desses pacientes ${ }^{8,11-13}$, sendo observado que a qualidade de vida é parâmetro que pode estar relacionado a aspectos demográficos, clínicos e terapêuticos e que a satisfação com a vida é uma percepção individual ${ }^{8}$.

Tendo em vista o relatado, propôs-se este trabalho que visou comparar a QSV do paciente antes da sua internação na UTI com a QSV 7, 90 e 180 dias após a alta hospitalar. Secundariamente, foram avaliados quais aspectos demográficos, clínicos e terapêuticos relacionaram-se com a QSV do paciente após a alta hospitalar.

\section{MÉTODO}

Após a aprovação pelo Comitê de Ética em Pesquisa em Seres Humanos da Universidade Federal de Santa Catarina, foi realizado um estudo de coorte prospectivo, não controlado, com abordagens qualitativa e quantitativa. Foram incluídos no estudo todos os pacientes que receberam alta da UTI do Hospital Universitário da UFSC, no período de maio a julho de 2005, maiores de 18 anos, que permaneceram por mais de 24 horas internados nessa unidade e dos quais, ou de seus representantes legais, foi obtido o consentimento livre e esclarecido. Foram excluídos os pacientes cujo motivo de admissão na UTI foi pós-operatório de intervenção cirúrgica eletiva e aqueles que foram transferidos para outros hospitais.

Os dados computados foram obtidos através de um questionário (Anexo 1) elaborado pelos pesquisadores. Foram anotados os aspectos demográficos, clínicos e terapêuticos. Foram também avaliados aspectos referentes à QSV dos pacientes, que foram adaptados, após tradução, do trabalho de Capuzzo e col. ${ }^{12}$, sendo que eles autorizaram as adaptações necessárias. $\mathrm{O}$ questionário foi validado em projeto piloto que avaliou uma população em condições semelhantes à deste estudo e que foi realizado no período de dezembro de 2004 a abril de 2005.

Este estudo foi dividido em quatro etapas. Inicialmente, nas primeiras 24 horas da admissão na UTI, foram anotados os dados referentes aos aspectos clínicos, demográficos, e foram obtidos, do paciente 
ou do seu representante legal, dados que permitiram a avaliação da QSV do paciente nos últimos três meses que antecederam a sua internação na UTI. Quanto à qualidade de vida, o questionário apresentava uma escala de atividades em ordem decrescente de complexidade, sendo que o respondedor assinalava a atividade mais complexa que o paciente era capaz de executar. Essa escala comparava as habilidades físicas, os hobbies e a situação laborativa dos diversos momentos do mesmo paciente, e não dos pacientes entre si. Para a avaliação da satisfação de vida, foi apresentada uma escala de satisfação pessoal para que o paciente a graduasse entre o totalmente satisfeito até o insatisfeito com a sua vida, no momento da entrevista. Ao final dos questionamentos, o pesquisador o grau de qualidade de vida global do paciente, baseado em uma escala de limitação física para a idade (Anexo 1).

Posteriormente, na primeira semana após a alta hospitalar e após três e seis meses, foram novamente coletados os dados referentes à QSV do paciente, naqueles momentos. Um dos pesquisadores entrou em contato, por telefone, com o paciente e/ou com o seu familiar, questionando sempre o mesmo respondedor inicial, sendo as perguntas direcionadas de maneira semelhante àquelas efetuadas na admissão na UTI. Ao final do estudo os questionários relativos à qualidade de vida após a alta hospitalar foram classificados como melhor/igual ou pior em relação ao momento prévio à internação. Esta classificação considerou as diferentes capacidades do mesmo paciente em realizar as tarefas descritas. Quanto à satisfação de vida, os questionários respondidos após a alta hospitalar foram comparados com o do momento prévio à internação e classificados como meIhor/igual ou pior satisfação pessoal de vida de cada paciente. Para as variáveis quantitativas foi utilizado o teste $t$ de Student e para as variáveis qualitativas o teste Qui-quadrado, sendo considerado significativo o valor de $p \leq 0,05$.

\section{RESULTADOS}

No período de maio a julho de 2005, foram internados na UTI do HU-UFSC, noventa e nove pacientes. Sessenta e oito preencheram os critérios para a inclusão no estudo, 34 foram a óbito, um não aceitou participar do estudo e seis foram transferidos para a UTI de outros hospitais. $\mathrm{Na}$ alta hospitalar 27 pacientes responderam ao questionário. Após três meses, um paciente foi a óbito, e não foi possível contatar outros quatro. Após seis meses, não foi possível contatar com um paciente. Portanto, completaram o estudo 21 pacientes.

Os questionários foram respondidos em $66,66 \%$ dos casos pelos pacientes e em $33,33 \%$ por parentes próximos.

$\mathrm{Na}$ tabela 1 pode ser observada a relação entre as variáveis clínicas e demográficas dos pacientes estudados e a sua qualidade de vida após 7, 90 e 180 dias da alta hospitalar. Essas mesmas variáveis relacionadas à satisfação de vida dos pacientes são destacadas na tabela 2.

A comparação entre a QSV dos pacientes antes da internação na UTI e após 7, 90 e 180 dias da alta hospitalar está demonstrada nas figuras 1 e 2 .

Tabela 1 - Relação entre as Variáveis Clínicas e Demográficas dos Pacientes e a sua Qualidade de Vida 7, 90 e 180 Dias após a Alta Hospitalar.

\begin{tabular}{|c|c|c|c|c|c|c|c|c|c|}
\hline \multirow[t]{2}{*}{ Variáveis Clínicas e Demográficas } & \multicolumn{2}{|c|}{$\begin{array}{l}\text { Qualidade de Vida } \\
\text { (7 dias da alta) } \\
n=21\end{array}$} & \multirow[t]{2}{*}{$\mathrm{p}$} & \multicolumn{2}{|c|}{$\begin{array}{c}\text { Qualidade de Vida } \\
\text { (90 dias da alta) } \\
n=21\end{array}$} & \multirow[t]{2}{*}{$\mathrm{p}$} & \multicolumn{2}{|c|}{$\begin{array}{c}\text { Qualidade de Vida } \\
\text { (180 dias da alta) } \\
n=21\end{array}$} & \multirow[t]{2}{*}{$\mathrm{p}$} \\
\hline & $\begin{array}{l}\text { Melhor } \\
\text { Igual }\end{array}$ & Pior & & $\begin{array}{l}\text { Melhor } \\
\text { Igual }\end{array}$ & Pior & & $\begin{array}{l}\text { Melhor } \\
\text { Igual }\end{array}$ & Pior & \\
\hline Idade média (anos) $^{\star}$ & 51,3 & 52,6 & NS & 56,4 & 41 & $p<0,05$ & 56,4 & 41 & $\mathrm{p}<0,05$ \\
\hline Sexo $(M: F)^{\dagger}$ & $8: 2$ & $8: 3$ & NS & $11: 4$ & $5: 1$ & NS & $11: 4$ & $5: 1$ & NS \\
\hline Escolaridade (anos) ${ }^{*}$ & 8,1 & 10,3 & NS & 9,8 & 7,8 & NS & 9,8 & 7,8 & NS \\
\hline $\begin{array}{l}\text { Tempo médio de internação na UTI } \\
\text { (dias)* }^{*}\end{array}$ & 5,2 & 6,4 & NS & 5,9 & 5,7 & NS & 5,9 & 5,7 & NS \\
\hline $\begin{array}{l}\text { Tempo médio de internação no hos- } \\
\text { pital (dias)* }\end{array}$ & 18,5 & 30,5 & NS & 27,1 & 19 & NS & 27,1 & 19 & NS \\
\hline
\end{tabular}

* Variável em que foi utilizado o teste $t$ de Student, sendo considerado significativo $p \leq 0,05$. NS = sem estatística significativa

† Variável em que foi utilizado o teste Qui-quadrado, sendo considerado significativo $\mathrm{p} \leq 0,05$. 
Tabela 2 - Relação entre as Variáveis Clínicas e Demográficas dos Pacientes e a sua Satisfação de Vida 7, 90 e 180 Dias após a Alta hospitalar

\begin{tabular}{|c|c|c|c|c|c|c|c|c|c|}
\hline \multirow[t]{2}{*}{ Variáveis Clínicas e Demográficas } & \multicolumn{2}{|c|}{$\begin{array}{c}\text { Satisfação } \\
\text { com a Vida } \\
\text { (7 dias da alta) }\end{array}$} & \multirow[t]{2}{*}{$\mathrm{p}$} & \multicolumn{2}{|c|}{$\begin{array}{c}\text { Satisfação } \\
\text { com a Vida } \\
\text { (90 dias da alta) }\end{array}$} & \multirow[t]{2}{*}{$p$} & \multicolumn{2}{|c|}{$\begin{array}{c}\text { Satisfação } \\
\text { com a Vida } \\
\text { (180 dias da alta) }\end{array}$} & \multirow[t]{2}{*}{$\mathrm{p}$} \\
\hline & Melhor/lgual & Pior & & Melhor/Igual & Pior & & Melhor/lgual & Pior & \\
\hline Idade média (anos)* & 51,4 & 54,5 & NS & 53,3 & 39,5 & NS & 51,8 & 53,5 & NS \\
\hline Sexo $(M: F)^{\dagger}$ & $13: 4$ & $3: 1$ & NS & $14: 5$ & $2: 0$ & NS & $14: 5$ & $2: 0$ & NS \\
\hline Escolaridade (anos) ${ }^{*}$ & 9,4 & 8,5 & NS & 9,7 & 5 & NS & 8,7 & 14 & NS \\
\hline Índice APACHE II médio* & 16,9 & 15,3 & NS & 16,4 & 18,5 & NS & 16,9 & 13,5 & NS \\
\hline $\begin{array}{l}\text { Tempo médio de internação na UTI } \\
\text { (dias)* }\end{array}$ & 6,4 & 3,5 & NS & 6,1 & 3,5 & NS & 6,1 & 3 & NS \\
\hline $\begin{array}{l}\text { Tempo médio de internação hospi- } \\
\text { tal (dias)* }\end{array}$ & 25,2 & 22,8 & NS & 25,8 & 14,5 & NS & 25,6 & 16,5 & NS \\
\hline
\end{tabular}

* Variável em que foi utilizado o teste $t$ de Student, sendo considerado significativo $p \leq 0,05$.

NS = sem estatística significativa

† Variável em que foi utilizado o teste Qui-quadrado, sendo considerado significativo $p \leq 0,05$.

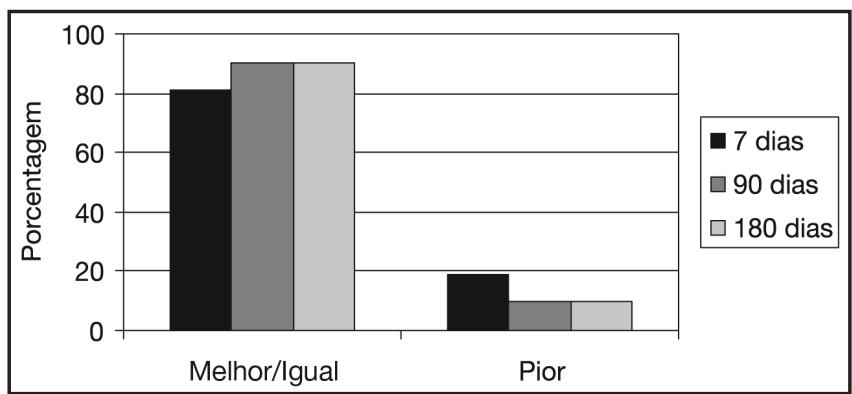

Figura 1 - Comparação entre a Satisfação de Vida dos Pacientes Antes da Internação na UTI e Após 7, 90 e 180 Dias da Alta Hospitalar. $\mathrm{N}=21$.

Teste Qui-quadrado sem significância estatística, sendo significativo $p \leq 0,05$

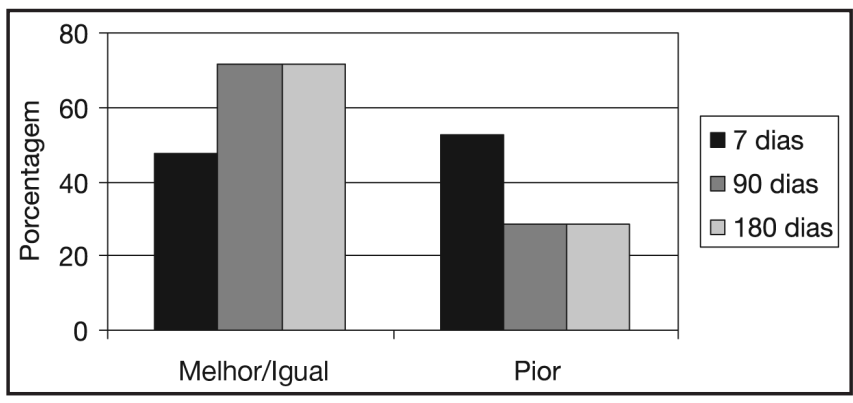

Figura 2 - Comparação entre a qualidade de vida dos pacientes antes da internação na UTI e após 7, 90 e 180 dias da alta hospitalar. $\mathrm{N}=21$.

${ }^{\star}$ Teste Qui-quadrado sem significância estatística, sendo significativo $p \leq 0,05$

Tabela 3 - Relação entre a Qualidade de Vida dos Pacientes após a Alta Hospitalar e a Terapêutica Instituída durante a Internação na UTI.

\begin{tabular}{|c|c|c|c|c|c|c|}
\hline \multirow{2}{*}{$\begin{array}{l}\text { Qualidade } \\
\text { de Vida }\end{array}$} & \multicolumn{2}{|c|}{7 dias após a alta } & \multicolumn{2}{|c|}{90 dias após a alta } & \multicolumn{2}{|c|}{180 dias após a alta } \\
\hline & $\begin{array}{c}\text { Fármaco } \\
\text { Vasoativo* }\end{array}$ & $\begin{array}{l}\text { Ventilação } \\
\text { Mecânica* }\end{array}$ & $\begin{array}{l}\text { Fármaco } \\
\text { Vasoativo* }\end{array}$ & $\begin{array}{l}\text { Ventilação } \\
\text { Mecânica* }\end{array}$ & $\begin{array}{l}\text { Fármaco } \\
\text { Vasoativo* }\end{array}$ & $\begin{array}{l}\text { Ventilação } \\
\text { Mecânica* }\end{array}$ \\
\hline Melhor/igual & $5(n=10)$ & $4(n=10)$ & $5(n=15)$ & $8(n=15)$ & $5(n=15)$ & $8(n=15)$ \\
\hline Pior & $4(n=11)$ & $7(\mathrm{n}=11)$ & $4(n=6)$ & $3(n=6)$ & $4(n=6)$ & $3(n=6)$ \\
\hline$p$ & NS & NS & NS & NS & NS & NS \\
\hline
\end{tabular}

* Variável em que foi usado o teste Qui-quadrado, sendo considerado significativo $p \leq 0,05$.

NS = sem estatística significativa

Tabela 4 - Relação entre a Satisfação de Vida dos Pacientes após a Alta Hospitalar e a Terapêutica Instituída durante a Internação na UTI.

\begin{tabular}{|c|c|c|c|c|c|c|}
\hline \multirow{2}{*}{$\begin{array}{c}\text { Satisfação } \\
\text { de Vida }\end{array}$} & \multicolumn{2}{|c|}{7 dias após a alta } & \multicolumn{2}{|c|}{90 dias após a alta } & \multicolumn{2}{|c|}{180 dias após a alta } \\
\hline & $\begin{array}{c}\text { Fármaco } \\
\text { Vasoativo* }\end{array}$ & $\begin{array}{l}\text { Ventilação } \\
\text { Mecânica* }\end{array}$ & $\begin{array}{c}\text { Fármaco } \\
\text { Vasoativo * }\end{array}$ & $\begin{array}{l}\text { Ventilação } \\
\text { Mecânica* }\end{array}$ & $\begin{array}{c}\text { Fármaco } \\
\text { Vasoativo * }\end{array}$ & $\begin{array}{l}\text { Ventilação } \\
\text { Mecânica* }\end{array}$ \\
\hline Melhor/igual & $7(n=17)$ & $9(\mathrm{n}=17)$ & $8(n=19)$ & $10(n=19)$ & $8(n=19)$ & $11(n=19)$ \\
\hline Pior & $2(n=4)$ & $2(n=4)$ & $1(n=2)$ & $1(n=2)$ & $1(n=2)$ & $0(n=2)$ \\
\hline $\mathrm{p}$ & NS & NS & NS & NS & NS & NS \\
\hline
\end{tabular}

* Variável em que foi usado o teste Qui-quadrado, sendo considerado significativo $p \leq 0,05$.

NS= não significativo 
Nas tabelas 3 e 4, foram analisados, em relação à QSV dos pacientes, o uso ou não de ventilação mecânica e a necessidade da administração de fármacos vasoativos.

Ao ser avaliado o percentual de pacientes que trabaIhavam anteriormente à internação, observou-se que dos 21 pacientes estudados, 15 exerciam alguma atividade laborativa fora de casa. Dos que retomaram suas atividades laborativas após a alta hospitalar, 2 pacientes $(13,3 \%)$ retornaram ao emprego na primeira semana após a alta, 7 pacientes $(46,7 \%)$ retornaram ao emprego após 90 dias e 9 (60\%) após 180 dias.

\section{DISCUSSÃO}

A insuficiência de leitos nas UTI, o alto custo financeiro e emocional do tratamento dos pacientes gravemente enfermos e a necessidade de racionalização dos leitos nessas unidades tem levado à necessidade de avaliação da QSV desses pacientes ${ }^{16-19}$. Entretanto, esse tema ainda é pouco debatido no meio médico. Cita-se como exemplo o trabalho de Cuthbertson e col. ${ }^{16}$, em cujos dados foi apontado que apenas $2 \%$ dos artigos publicados sobre UTI avaliaram a QSV dos pacientes. Este trabalho utilizou uma adaptação do trabalho de Capuzzo e col. ${ }^{12}$, onde os autores avaliaram as atividades diárias, a capacidade física e a satisfação subjetiva de saúde e felicidade dos pacientes e constataram que questionários curtos, objetivos e simples de responder são tão sensíveis para a avaliação da QSV quanto os questionários extensos, além de serem mais facilmente aplicáveis aos pacientes.

Neste estudo observou-se que a qualidade de vida dos pacientes que receberam alta da UTI, apresentou um decréscimo na primeira semana da alta hospitalar, situação esta que se inverteu na avaliação de 90 dias e que se manteve na avaliação de 180 dias. Resultados semelhantes são descritos na literatura ${ }^{8,13,15-21,24}$. Esse fato pode ser explicado pelo tempo que é necessário para que o ser humano readquira as suas funções motoras e habilidades prévias e se adapte à uma nova realidade.

O indicador não biológico melhor aceito para a verificação do nível de saúde é o retorno ao trabalho, que sofre a influência da idade, dos fatores sócio-econômicos e do tipo de emprego ${ }^{21}$. Os resultados deste estudo apontaram que a maioria dos pacientes retornou ao seu emprego 180 dias após a alta hospitalar. No estudo de Frick e col. ${ }^{13}$, 58\% dos pacientes retornaram às suas atividades laborativas. Entretanto, os resultados foram avaliados após um ano da alta hospitalar, assim como no trabalho de Cuthbertson e col. ${ }^{16}$. Nos estudos de Hurel e col..$^{22}$, Kvale e col. ${ }^{19}$ e de Lizana e col. ${ }^{21}$, resultados semelhantes foram obtidos respectivamente, após 6 meses, 7-8 meses e 18 meses da alta da UTI. Um dado curioso observado neste estudo é que dos nove pacientes que retornaram ao trabalho após 180 dias, um pertencia ao grupo que referia piora da satisfação com a vida com melhora da qualidade de vida. Pode-se inferir que a idade (48 anos) e a profissão (autônomo) desse paciente tenham influenciado nesse resultado, pois ele possuía elevada expectativa de recuperação.

É relatado que os pacientes, mesmo percebendo um decréscimo na sua qualidade de vida ao receberem alta hospitalar, sentem-se satisfeitos. Essa satisfação é demonstrada quando esses pacientes referem que, caso fosse necessário, aceitariam ser novamente internados na UTI $22-24$. No presente estudo, bem como no de Montuclard e col. ${ }^{23}$ mais de $80 \%$ dos pacientes referiram satisfação. Resultados discretamente superiores foram apontados por Wehler e col. ${ }^{24}$. Merece destaque o fato de que os pacientes sentiram-se satisfeitos mesmo apresentando comprometimento na qualidade de vida. Eddleston e col. ${ }^{15}$ mostraram que os pacientes, apesar de referirem fadiga, pequeno poder de concentração e distúrbios do sono, sentiam-se satisfeitos. Nesse mesmo estudo foi apontado que esses sintomas desapareceram após um ano da alta hospitalar. No trabalho de Kaarlola e col. ${ }^{20}$ a avaliação foi realizada após seis anos, e os autores demonstraram que $91 \%$ dos pacientes sentiam-se satisfeitos com sua vida. O mais importante para o paciente é a felicidade de estar novamente no seio da sua família e a sensação da superação de um grande obstáculo. Diante do exposto, pode-se inferir que o ser humano deseja, primordialmente, estar vivo. É importante ressaltar que, neste trabalho, a satisfação com a vida tendeu a permanecer, mesmo após o paciente ter sido introduzido novamente à sua rotina diária.

Neste estudo a idade dos pacientes não influenciou na satisfação das suas vidas. Resultados semelhantes são apontados na literatura ${ }^{15,23}$. Entretanto, outros autores mostraram que os pacientes mais idosos apresentaram pior qualidade de vida após a alta hospitalar ${ }^{7,8}$. Dowdy e col. ${ }^{25}$ demonstraram que os pacientes mais velhos apresentavam menor qualidade de vida. Granja e col. ${ }^{8}$ relataram que os pacientes com mais 
idade apresentavam mais dor e desconforto e que a piora da qualidade de vida estaria mais relacionada à causa da internação na UTI e a baixa qualidade de vida prévia, do que à idade dos pacientes. Neste trabalho a satisfação de vida dos pacientes não mostrou relação com o tempo de internação na UTI. Resultados semelhantes foram anteriormente descritos ${ }^{16,26,27}$.

Kvale e col. ${ }^{18}$ mostraram que não existe correlação entre a gravidade do quadro clínico (APACHE II) e a QSV dos pacientes. Os resultados deste estudo corroboram com essa afirmação. Entretanto, outros estudos apontam resultados divergentes ${ }^{8,16}$.

É descrito que os pacientes com pior prognóstico consomem mais os recursos oferecidos pela UTI ${ }^{21}$. As UTI são de fundamental importância para pacientes gravemente enfermos; entretanto, há uma parcela de pacientes que não se beneficiará dos serviços desse setor. A dificuldade de ser estabelecida qual a parcela que irá ou não se beneficiar do tratamento intensivo é um desafio, pois não existem dados suficientemente sensíveis e específicos que possam predizer a mortalidade e a morbidade dos pacientes, após alta dessas unidades ${ }^{21,22}$. Hofhuis e col. ${ }^{14}$ demonstraram que a qualidade de vida prévia à internação nas UTI tem um impacto significante na mortalidade e na qualidade de vida após a alta dessas unidades. Neste estudo pode-se constatar que os pacientes sentiram-se satisfeitos após a alta da UTI, e que a sua qualidade de vida melhorou com o passar do tempo. Deve ser ressaltado que o número de pacientes estudados foi pequeno, e que o período do estudo foi limitado, o que pode ser um viés para os resultados.

Quanto às implicações deste estudo para a prática clínica e pesquisa científica, o conhecimento das mudanças da qualidade de vida a curto e longo prazo poderão fornecer, ao médico intensivista e à equipe multidisciplinar da UTI, informações úteis para futuras decisões. Por esse motivo, os autores deste estudo se permitem sugerir que haja a sua continuidade e que estudos semelhantes sejam realizados em outros centros.

Diante dos resultados apontados pode-se concluir que houve uma tendência de os pacientes que receberam alta da UTI-HU apresentarem uma QSV melhor/igual. Enquanto a satisfação de vida desses pacientes permaneceu semelhante após 90 e 180 dias da alta, houve melhora significativa da qualidade de vida. Os pacientes que apresentaram satisfação de vida melhor/ igual foram aqueles que permanecerem mais tempo internados na UTI ou no hospital. Não houve relação entre a QSV e a terapêutica instituída. A idade dos pacientes mostrou influência direta na sua qualidade de vida após 90 dias da alta hospitalar. Após 180 dias da alta hospitalar, $60 \%$ dos pacientes havia retornado ao trabalho.

\section{ANEXO 1 - FICHA DE COLETA DE DADOS}

Data

Protocolo

Prontuário

Nome

Idade

Endereço

Telefone

Sexo (_) Masculino (_) Feminino

Respondedor (_) Paciente (_) Familiar

Tempo de Internação em dias Hospital (_) UTI (_)

Ventilação Mecânica (_) Não (_) Sim

APACHE II escore

ATIVIDADE FÍSICA (Admissão) (7 dias) (90 dias) (180 dias)

$7^{\circ}$ ) Desenvolve atividade de trabalho fora de casa (_)

$\left(\_\right)\left(\_\right)\left(\_\right)$

$\left.6^{\circ}\right)$ Sobe uma rampa de escada sem fadiga $\left(\_\right)\left(\_\right)\left(\_\right)$ $\left(\_\right)$

$\left.5^{\circ}\right)$ Vai fazer compras e segura as sacolas $\left(\_\right)\left(\_\right)\left(\_\right)\left(\_\right)$

$\left.4^{\circ}\right)$ Sai a passeio $\left(\_\right)\left(\_\right)\left(\_\right)\left(\_\right)$

$\left.3^{\circ}\right)$ Faz as atividades domésticas $\left(\_\right)\left(\_\right)\left(\_\right)\left(\_\right)$

$\left.2^{\circ}\right)$ Lava-se e se veste $\left(\_\right)\left(\_\right)\left(\_\right)\left(\_\right)$

$\left.1^{\circ}\right)$ Está acamado $\left(\_\right)\left(\_\right)\left(\_\right)\left(\_\right)$

PSICOSSOCIAL: COMO TRANSCORRE SEU TEMPO LIVRE?

$\left.5^{\circ}\right)$ Há hobbies: ocupa-se de horto/jardim ou esporte

(_) (_) (_) (_)

$\left.4^{\circ}\right)$ Freqüenta ou vê amigos (_) (_) (_) (_)

$\left.3^{\circ}\right)$ Lê jornal $\left(\_\right)\left(\_\right)\left(\_\right)\left(\_\right)$

$\left.2^{\circ}\right)$ Assiste à televisão $\left(\_\right)\left(\_\right)\left(\_\right)\left(\_\right)$

$\left.1^{\circ}\right)$ Freqüenta apenas os parentes $\left(\_\right)\left(\_\right)\left(\_\right)\left(\_\right)$

\section{SATISFAÇÃO DE VIDA:}

Quanto está satisfeito com sua vida?

Totalmente (_) (_) (_) (_)

Muito $\left(\_\right)\left(\_\right)\left(\_\right)\left(\_\right)$

Razoavelmente $\left(\_\right)\left(\_\right)\left(\_\right)\left(\_\right)$

Pouco $\left(\_\right)\left(\_\right)\left(\_\right)\left(\_\right)$

Insatisfeito $\left(\_\right)\left(\_\right)\left(\_\right)\left(\_\right)$ 


\section{QUALIDADE DE VIDA GLOBAL (avaliação subjetiva do pesquisador)}

$\left.1^{\circ}\right)$ Nenhuma limitação para a idade $\left(\_\right)\left(\_\right)\left(\_\right)\left(\_\right)$

$2^{\circ}$ ) Modesta limitação para a idade $\left(\_\right)\left(\_\right)\left(\_\right)\left(\_\right)$

$\left.3^{\circ}\right)$ Grave limitação $\left(\_\right)\left(\_\right)\left(\_\right)\left(\_\right)$

$\left.4^{\circ}\right)$ Totalmente dependente $\left(\_\right)\left(\_\right)\left(\_\right)\left(\_\right)$

\section{REFERÊNCIAS}

01. Moritz RD - A Morte e o Morrer nas Unidades de Terapia Intensiva, em David CM - Medicina Intensiva. Rio de Janeiro. Revinter, 2004;68-78.

02. Vincent $\mathrm{JL}$ - European attitudes towards ethical problems in intensive care medicine: results of an ethical questionnaire. Intensive Care Med, 1990;16:256-264

03. Moritz, RD, Pamplona F - Avaliação da recusa ou suspensão do tratamento considerados fúteis ou inúteis. RBTI, 2003;15:40-45.

04. Knaus WA, Draper EA, Wagner DP - APACHE II: a severity of disease classification system. Crit Care Med. 1985;13:818-829.

05. Ferreira FL, Bota DP, Bross A - Serial evaluation of the SOFA score to predict outcome in critically ill patients. JAMA. 2001;286:1754-1758.

06. Alves CJ, Terzi RGG, Franco GPP et al - Comparação entre o Modelo UNICAMP II e o APACHE II em uma UTI Geral. RBTI, 2003;15:144-152.

07. Wehler M, Hadzionerovic D, Aljukic $E$ et al - Health-related quality of life of multiple organ dysfunction patients: changes and comparison with normative population data. Crit Care, 2002;6: 118-128.

08. Granja C, Teixeira-Pinto A, Costa-Pereira A - Quality of life after intensive care - evaluation with EQ-5D questionnaire. Intensive Care Med, 2002;28:898-907.

09. Jacobs CJ, van der Vliet JA, van Roozendaal MT et al - Mortality and quality of life after intensive care for critical illness. Intensive Care Med, 1998; $14: 217-220$.

10. Capuzzo M, Bianconi M, Contu $P$ et al - Survival and quality of life after intensive care. Intensive Care Med, 1996;22:947-953.

11. Vazquez Mata G, Rivera Fernandez R, Gonzalez Carmona A et al - Factors related to quality of life 12 months after discharge from an intensive care unit. Crit Care Med, 1992;20:1257-1262.

12. Capuzzo M, Grasselli C, Carrer S et al - Validation of two quality of life questionnaires suitable for intensive care patients. Intensive Care Med, 2000;26:1296-1303.

13. Frick S, Uehlinger DE, Zurcher Zenklusen RM - Assessment of former ICU patients' quality of life: comparison of different quality-of-life measures. Intensive Care Med, 2002;28:1405-1410.

14. Hofhuis J, Hautvast JL, Schrijvers AJ et al - Quality of life on admission to the intensive care: can we query the relatives? Intensive Care Med, 2003;29:974-979

15. Eddleston JM, White P, Guthrie E et al - Survival, morbidity, and quality of life after discharge from intensive care. Crit Care Med, 2000;28:22932299.

16. Cuthbertson BH, Scott J, Strachan M et al - Quality of life before and after intensive care. Anaesthesia, 2005;60:332-339.

17. Williams TA, Dobb GJ, Finn JC et al - Long-term survival from intensive care: a review. Intensive Care Med, 2005;31:1306-1315

18. Kvale R, Ulvik A, Flaatten H - Follow-up after intensive care: a single center study. Intensive Care Med, 2003;29:2149-2156.

19. Badia X, Diaz-Prieto A, Gorriz MT et al - Using the EuroQol-5D to measure changes in quality of life 12 months after discharge from an intensive care unit. Intensive Care Med, 2001;27:1901-1907.

20. Kaarlola A, Pettila V, Kekki P - Quality of life six years after intensive care. Intensive Care Med, 2003;29:1294-1299.

21. Garcia Lizana F, Peres Bota D, Cubber $M$ et al - Long-term outcome in ICU patients: what about quality of life? Intensive Care Med, 2003;29:1286-1293.

22. Hurel D, Loirat P, Saulnier F et al - Quality of life 6 months after intensive care: results of a prospective multicenter study using a generic health status scale and a satisfaction scale. Intensive Care Med, 1997;23:331-337.

23. Montuclard L, Garrouste-Orgeas M, Timsit JF et al - Outcome, functiona autonomy, and quality of life of elderly patients with a long-term intensive care unit stay. Crit Care Med, 2000;28: 3389-3395.

24. Wehler M, Martus $P$, Geise A et al - Changes in quality of life after medica intensive care. Intensive Care Med, 2001;27:154-159.

25. Dowdy DW, Eid MP, Sedrakyan A et al - Quality of life in adult survivors of critical illness: a systematic review of literature. Intensive Care Med, 2005;31:611-620.

26. Rellos K, Falagas ME, Vardakas KZ et al - Outcome of critically ill oldestold patients (aged 90 and older) admitted to the intensive care unit. J Am Geriatr Soc, 2006;54:110-114

27. Stricker $\mathrm{KH}$, Cavegn $\mathrm{R}$, Takala $\mathrm{J}$ et al - Does ICU length of stay influence quality of life? Acta Anaesthesiol Scand, 2005;49:975-983. 